\title{
MINIATURIZED SPECTROMETERS FOR BIOCHEMICAL ANALYSIS
}

\author{
Gaylin M. Yee ${ }^{1}$, Paul A. Hing ${ }^{2}$, Nadim I. Maluf ${ }^{1,3}$, Gregory T. A. Kovacs ${ }^{1}$ \\ ${ }^{1}$ Stanford University \\ Center for Integrated Systems, CISX-205 \\ Stanford, CA 94305-4075
}

${ }^{2}$ Applied Biosystems Division, Perkin-Elmer Corporation Foster City, CA 94404

\author{
${ }^{3}$ Lucas Novasensor \\ 1055 Misson Court \\ Fremont, CA 94539
}

\begin{abstract}
Miniaturized spectrometers were demonstrated by mounting micromachined diffraction gratings onto $\mathrm{CCD}$ imaging devices. Two implementations were tested: one for a high dispersion and sensitivity application, and the other for a low-cost consumer application. The first system showed a dispersion of 1.7 $\mathrm{nm} /$ pixel and a resolution of $74.4 \mathrm{~nm} / \mathrm{nm}$ for the bandwidth of interest. The free spectral range of the device was designed to be 400-800 nm for this particular application. The diffraction efficiency of the system is $63 \%$. The second, low-cost system demonstrated a dispersion and resolution of $2.55 \mathrm{~nm} /$ pixel and $69.8 \mathrm{~nm} / \mathrm{nm}$ respectively. Results are shown for their applications in biochemical analysis. In addition, further optimization was sought by adding micromachined lenses and creating specialized gratings to compress and shape the spectral signal.
\end{abstract}

\section{BACKGROUND}

Spectrometers detect the chemical composition of a substance by measuring its absorption or fluorescence spectra. Light from the sample passes through a diffraction grating and disperses into a continuum of wavelengths. The longer the wavelength, the greater its angle of diffraction. By examining the intensities of the various frequencies, the substances can be identified by matching to the known spectral signatures of the chemical components.

Conventional benchtop spectrometers involve a complex system of lenses and moving parts, and are thus bulky and costly. By applying micromachining techniques it is possible to produce a spectrometer drastically reduced in size and intricacy. In addition, these systems are less expensive and compact, allowing them to be easily arrayed for improved spectral range and/or resolution.

Previously integrated spectrometers involve those formed with planar waveguides [1] or Fabry-Perot etalons [2]. Those that involve a diffraction grating have implemented bulk micromachined channels [3], amorphous silicon detectors on a transparent substrate [4], or free-standing gratings on a substrate [5]. However, many of these implementations are relatively involved and difficult to implement in volume.
Two implementations of a more manufacturable miniature spectrometer were demonstrated. Both devices are formed by mounting a transmission grating directly over a charge-coupled detector (CCD) imager, as shown in Fig. 1. The fine grating pitch creates high dispersion angles, thus providing sufficient spectral image dispersion without a large projection distance.

The first implementation was for applications in the detection of fluorescent dyes used in DNA separation [6]. Thus, both high sensitivity and dispersion were important, and so necessitated the use of a Peltier cooled CCD detector of $512 \times 64$ pixels. The second implementation was designed for use in lowcost applications such as colorimetry. A graving with wider pitch was mounted above a low-cost, easily available consumer CCD. In both applications, an extemal lens was used for light collimation and higher resolution and efficiency.

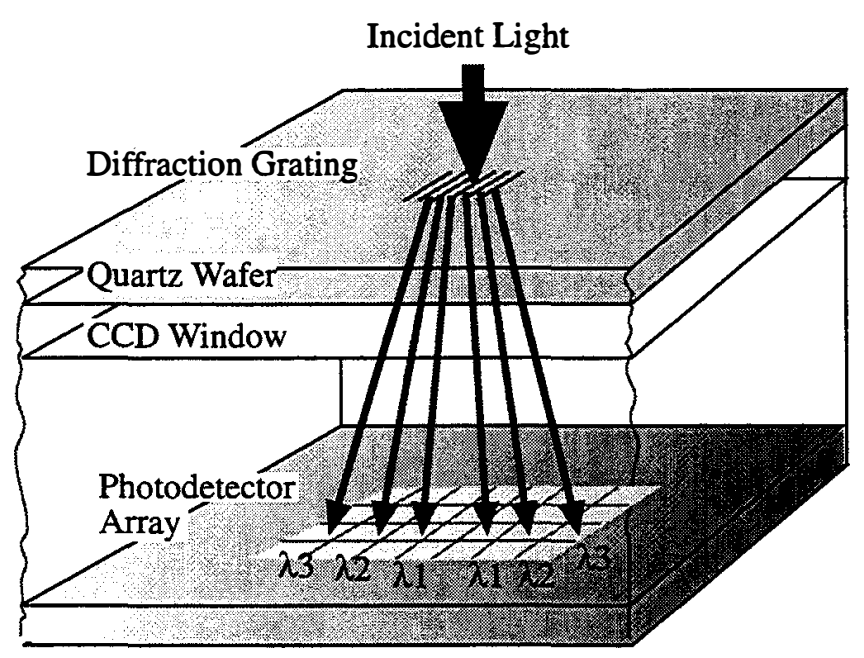

Figure 1: Cross-section of the spectrometer device. Light passes through the diffraction grating and disperses according to the wavelengths of their components, where $\lambda 3>\lambda 2>\lambda 1$. The spectral signals are recorded by the photodetector array for the determination of chemical substances. 


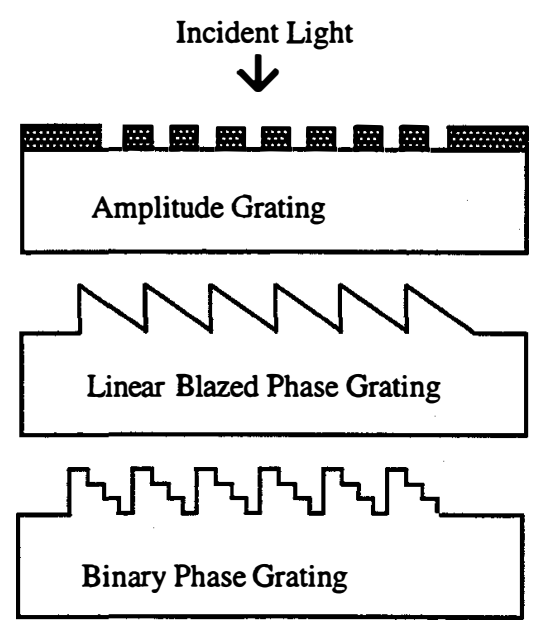

Figure 2: A schematic of basic grating types. Instead of blocking out sections of the light with opaque sections, phase gratings act to modulate its phase with variations in the grating shape. A binary step phase grating is a good approximation to the linear blaze, and is easily implemented with micromachining techniques.

\section{THEORY}

First order descriptions of diffraction gratings have long been available [7]. An amplitude grating consists of a simple array of slits, but has low efficiency in that a small percentage of light diffracts into the first peak, or spectral order. This can be remedied by using phase gratings or even blazed gratings, which have a theoretical efficiency of $100 \%$. A blazed grating can easily be approximated in micromachining technologies by etching a series of steps, as shown in Fig. 2.

Dispersion, or the amount of lateral distance change with respect to wavelength, for an amplitude grating with pitch $a$ is

$$
\left.D \equiv \frac{d x}{d \lambda}\right|_{\theta=\theta_{o}}=\frac{m \cdot h}{a \cdot \cos ^{3} \theta_{o}}
$$

where $m$ is the spectral order, $\theta_{o}$ is the angle of dispersion, and $h$ is the optical distance between the grating and the detector. Resolution, or the sharpness of the resolved peaks, is simply

$$
R \equiv \frac{\lambda}{\Delta \lambda}=\frac{N \cdot a \cdot \sin \theta_{o}}{\lambda}
$$

where $N$ is the number of lines illuminated. Also of importance is the free spectral range, or the range of wavelengths which the spectrometer can measure. Finally, the diffraction efficiency may be defined as the amount of light incident on the grating which is converted into useful signal. Efficiency of a phase modulated grating shaped into a number $I$ of binary steps is

$$
\eta=\left|\operatorname{sinc}\left[\frac{m}{I}\right] \frac{\operatorname{sinc}\left[m-\frac{\phi_{0}}{2 \pi}\right]}{\operatorname{sinc}\left[\frac{m-\frac{\phi_{o}}{2 \pi}}{I}\right]}\right|^{2}
$$

where $\lambda_{0}$ is the wavelength designed for and $\lambda$ is the actual wavelength used [7].

It has been shown [8] that ideal Fourier response of the overall system can be achieved if the light source is imaged onto the detector array. This was accomplished by placing the detector in the focal plane of a small lens. As a result, the resolution and the free spectral range were improved.

\section{FABRICATION}

The gratings were fabricated using chrome on quartz wafers. Because of the high resolution 0.4-0.5 $\mu \mathrm{m}$ step dimensions, the PMMA resist was exposed using direct write on a Hitachi HL700 electron beam system. After wet etching the chrome, the underlying quartz was RIE etched in oxygen. These processes were repeated for multiple steps in blazed gratings.

For the first implementation, a grating with $0.4 \mu \mathrm{m}$ slit width and $0.8 \mu \mathrm{m}$ pitch was mounted on the CCD top window, approximately $5.7 \mathrm{~mm}$ above the detector. The CCD consists of $512 \times 64$ pixels, each $27 \times 27 \mu \mathrm{m}$ in size. In the low-cost implementation, a grating of $0.5 \mu \mathrm{m}$ slits and $1.0 \mu \mathrm{m}$ pitch was mounted over a consumer grade CCD camera, QuickCam ${ }^{\mathrm{TM}}$ (from Connectix Corp., San Mateo, CA). The detector consisted of $320 \times 240$ pixels, each $11 \times 10.4 \mu \mathrm{m}$.

\section{EXPERIMENTAL RESULTS}

The setup for the detection of DNA labeling dyes is shown in Fig. 3. The sample is excited using a laser source and the resulting fluorescence is focused onto the spectrometer. The spectra are shown in Fig. 4 showing the response of the system to the $\mathrm{He}-\mathrm{Ne}$ laser and to a cyanine dye designed to emit at 657 $\mathrm{nm}$. Using these two wavelengths for calibration, the measured dispersion and resolution are $1.7 \mathrm{~nm} /$ pixel and $74.4 \mathrm{~nm} / \mathrm{nm}$ respectively. The free spectral range is $400-800 \mathrm{~nm}$. At short wavelengths, the response is limited by the CCD quantum efficiency; at longer wavelengths, it is limited by total internal reflection at the lower interface of the $C C D$ window. The range could easily be extended to longer wavelengths by altering the dimensions of the device. The diffraction efficiency of a phase graing for the system was measured to be $63 \%$.

In addition, further sensitivity was obtained by adding a micromachined cylindrical lens on top of the grating. The lens was a binary 3-phase Fresnel zone plate which focused light in the direction perpendicular to that of the dispersion. As shown in Fig. 5, this increased the signal/noise ratio of the signal while requiring a narrower and less expensive photodetector

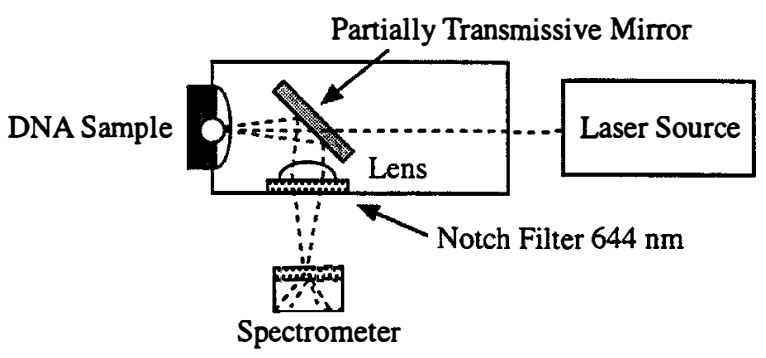

Figure 3: Detection setup involving sample, light source and spectrometer. A laser excites the sample and fluoresces its dyes. The resulting light is reflected by the mirror into a lens which focuses the light onto the spectrometer. 


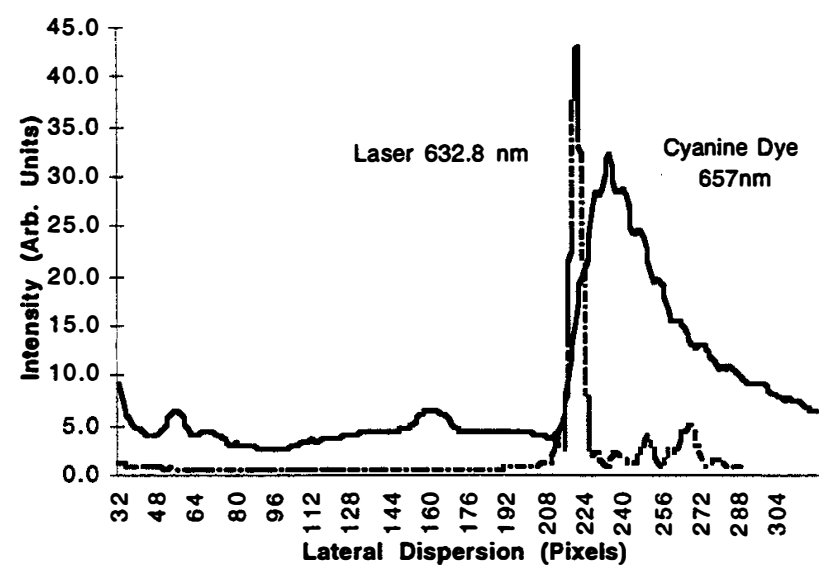

Figure 4: The response of the spectrometer showing the presence and intensity of the cyanine dye. A He-Ne laser spectra is provided for comparison.

array. This is especially critical to increase the response and perhaps allow the use of a less expensive, non-cooled device.

The low-cost implementation was tested with the biochemical fluorescent dye known as fluorescein. The responses in two different solvents are shown as in Fig. 6. The dispersion was measured as $2.55 \mathrm{~nm} /$ pixel and the resolution as $69.8 \mathrm{~nm} / \mathrm{nm}$ for the bandwidth of interest. The free spectral range is $500-800 \mathrm{~nm}$, demonstrating less sensitivity at low frequencies and a cutoff due to CCD dimensions for the high frequencies. The results for both spectrometers are summarized in Table 1.

In the assembly of these devices, little attention was given to grating alignment, interface imperfections, and glass irregularities. These normally result in a loss of resolution. This problem will be remedied in the future by aligning the glass to the detector at the wafer level and using anodic bonding. Loss of efficiency was also observed (Table 1) and is attributed to decreased transmission through the glass at high diffraction angles and to lack of depth control in the etch of the gratings. These undesirable effects will be alleviated by improving process monitoring and characterization.

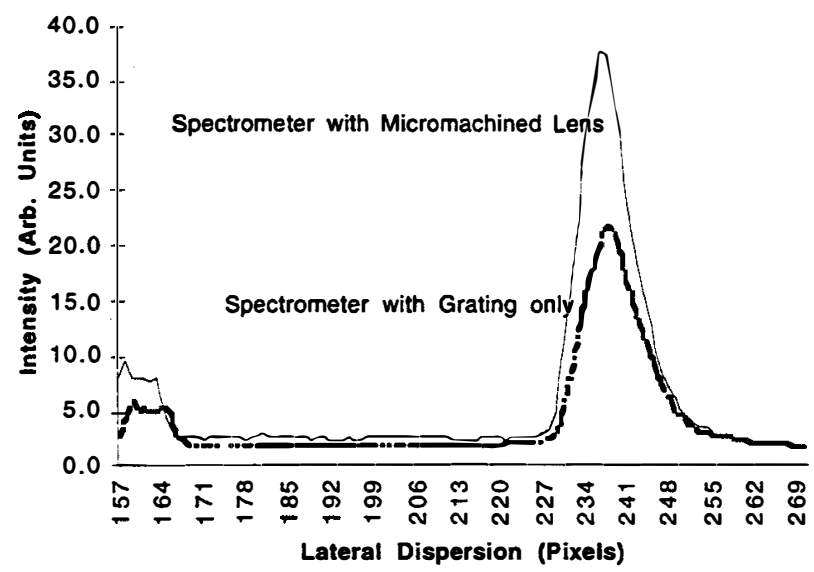

Figure 5: The improved signal from the more sensitive spectrometer with a micromachined cylindrical Fresnel lens. The lens was shaped using a three phase step blaze in order to focus the dispersing light into a single line.

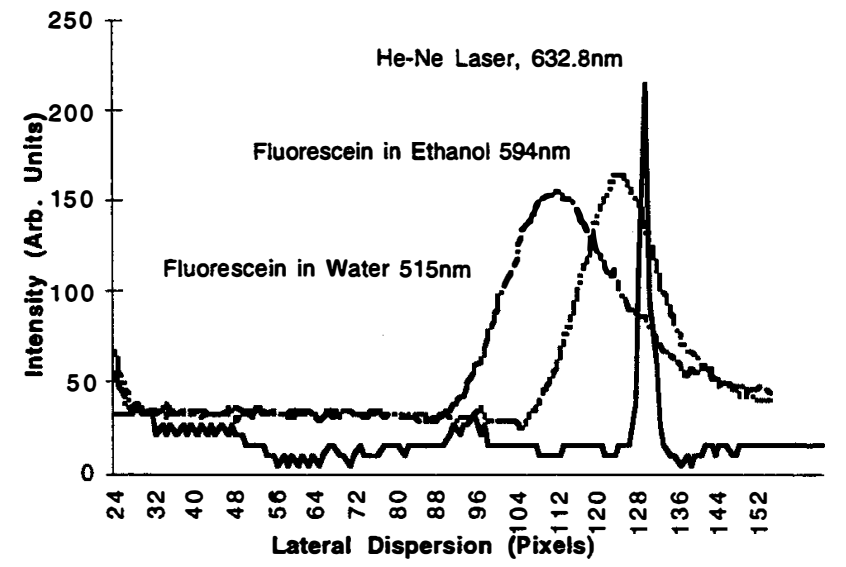

Figure 6: Response of the low cost spectrometer to the dye fluorescein. The spectrometer clearly distinguishes the peaks of the dye in water versus ethanol. A He-Ne laser spectrum is provided for comparison.

\section{SPECTRAL DETECTION USING CUSTOMIZED GRATINGS}

The overall spectrometer system was further optimized by using custom gratings to shape the spectral signal. Earlier research in such masks has mainly focused on improving the imaging in optical lithography [9]. Here, the process was extended to provide expanded resolution in the spectrometry of biochemicals in a specific bandwidth of $550 \mathrm{~nm}-660 \mathrm{~nm}$. Since only the first order spectral peaks on each side provide information, these gratings were designed to compress central "dead space" between the first order peaks. This was done by simulating the diffraction process operating in reverse and dithering the mask pixels to provide the desired response. Shown in Fig. 7 is a SEM micrograph of the resultant mask of $512 \times 512$ pixels of $0.4 \mu \mathrm{m}$ width.

Results obtained using the nonlinear grating are shown in Fig. 8. With a source at $635 \mathrm{~nm}$, the diffraction peaks for the conventional grating are $10.64 \mathrm{~mm}$ apart while those of the nonlinear grating are only $3.54 \mathrm{~mm}$ apart. This is nearly a third of that of the conventional grating, providing substantial savings in area. However, this reduction is at the cost of a loss of both the efficiency and local dispersion.

\section{CONCLUSIONS AND FUTURE WORK}

An easily manufacturable spectrometer has been demonstrated for use in biochemical analysis. The obtained dispersions and resolutions were sufficient to detect the

\begin{tabular}{lcccc} 
Results for & the & \multicolumn{2}{c}{ High-Dispersion } & Spectrometer \\
\hline & Theory & Actual & \\
\hline Dispersion & 1.5 & 1.7 & $\mathrm{~nm} / \mathrm{pixel}$ \\
Resolution & 113 & 74.4 & $\mathrm{~nm} / \mathrm{nm}$ \\
Efficiency & 80.2 & 63 & $\%$ \\
Results for & the & Low-Cost & Spectrometer \\
Dispersion & 2.59 & 2.55 & $\mathrm{~nm} / \mathrm{pix}$ el \\
Resolution & 100 & 69.8 & $\mathrm{~nm} / \mathrm{nm}$ \\
\hline
\end{tabular}

Table 1: Summary of Results for both implementations of the spectrometer. 
difference of several biochemical dyes. In addition, we have optimized the response by using either additional micromachined cylindrical lenses or custom, nonlinear gratings to shape the resulting speckal signal.

Future work will concentrate on an entirely integrated system, which will allow for scaling and manufacturability. The device will consist of a micromachined grating anodically bonded onto a CMOS imager. The alignment would be at the wafer level, providing improved alignment and tilt which in turn, will enhance resolution and dispersion.

\section{ACKNOWLEDGMENTS}

The authors would like to thank the Applied Biosystems Division of Perkin-Elmer and the National Science Foundation for supporting this research (Kovacs' NYI Award, Grant \# ECS9358289, and Yee's Graduate NSF Fellowship). In addition, thanks go to Mark McCord, Chris Storment, Mike Albin; Kristen Pol, and Hao $\mathrm{Xu}$ for technical assistance and support. The e-beam system is also supported by a grant from the National Science Foundation.

\section{REFERENCES}

1. D. Goldman, P. White, N. Anheier, "Miniaturized Spectrometer Employing Planar Waveguides and grating Couplers for Chemical Analysis", Applied Optics, Vol.29, No. 31, (November 1990), pp. 4583-4589.

2. J. Holm-Kennedy, K. Tsang, W. Sze, F. Jiang, D. Yang, "A Novel Monolithic, Chip Integrated, Color Spectrometer", SPIE, vol . 1527, (1991), pp. 322-331.

3. T. A. Kwa and R. F. Wolffenbuttel, "Integrated Grating/Detector Array Fabricated in Silicon Using Micromachining Techniques", Sensors and Actuators A, vol 31 (1992), pp. 259-266.

4. Z.E. Smith, III and R. Street, "Spectral Resolving and Sensing Apparatus”, U.S. Patent No. 5,037,201, 1991.

5. S.S. Lee, L.Y. Lin, and M. C. Wu, "Surface-Micromachined Free-space Micro-Optical Systems Containing ThreeDimensional Microgratings". Applied Physics Letters, vol.65, no. 15 , (1995), pp. 2135-2137.

6. M. O'Neill, "Sequencers Benefit from Solid-State Detectors", Laser Focus World, (October 1995), pp. 135-142.

7. M. Bass, Handbook of Optics, vol. II, New York: Optical Society of America, 1994), pp. 8.9-8.10.

8. J. W. Goodman, Introduction to Fourier Optics, (New York: McGraw-Hill, 1996), pp. 98-114, 210-214.

9. T. Terasawa, N. Hasegawa, H. Fukuda, S. Katagiri, "Imaging Characteristics of Multi-Phase-Shifting and Halftone PhaseShifting Masks", Journal of Applied Physics, (November 1991), pp. 2991-2996,

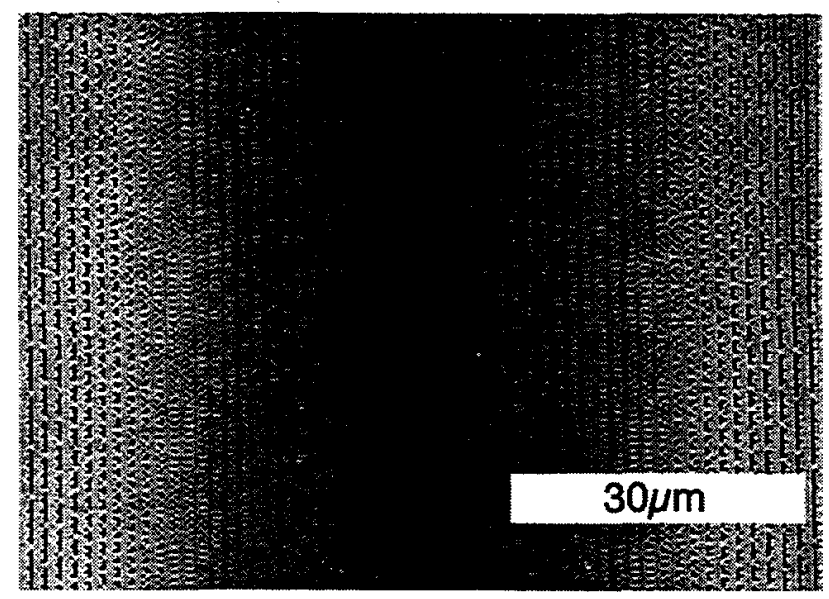

Figure 7: SEM photo of the computed custom grating, consisting of a $512 \times 512$ array of mask grating pixels $0.4 \times 0.4$ $\mu \mathrm{m}$ in size. The pixels are dithered vertically into those which are clear, chrome-covered, and etched. The diffracting light is dispersed horizontally.

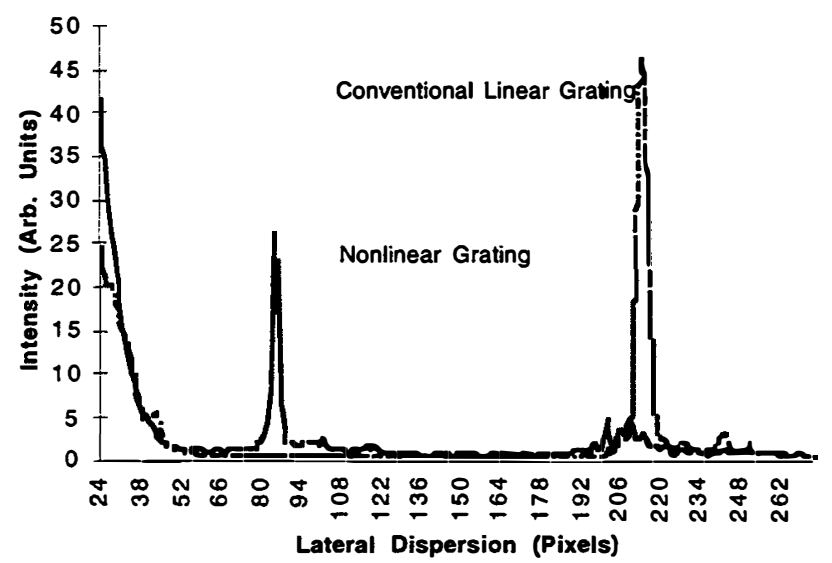

Figure 8: Comparison of the results of the customized nonlinear grating versus that of a standard linear grating. The first order peak has moved drastically towards the center, where a part of the incident beam is shown. Note the decreased amplitude, which denotes a loss of diffraction efficiency due to the large amount of mask pixels covered with chrome. 